Correction

\title{
Correction: Liu, W. et al. A Highly Sensitive Humidity Sensor Based on Ultrahigh-Frequency Microelectromechanical Resonator Coated with Nano-Assembled Polyelectrolyte Thin Films. Micromachines, 2017, 8, 116
}

\author{
Wenpeng Liu, Hemi Qu *, Jizhou Hu, Wei Pang, Hao Zhang and Xuexin Duan * \\ State Key Laboratory of Precision Measuring Technology \& Instruments, Tianjin University, \\ Tianjin 300072, China; liuwenpeng@tju.edu.cn (W.L.); mrhjz1222@tju.edu.cn (J.H.); \\ weipang@tju.edu.cn (W.P.); haozhang@tju.edu.cn (H.Z.) \\ * Correspondence: hemi.qu@tju.edu.cn (H.Q.); xduan@tju.edu.cn (X.D.); Tel.: +86-22-2740-1002 (H.Q. \& X.D.) \\ Academic Editor: Nam-Trung Nguyen \\ Received: 26 May 2017; Accepted: 30 May 2017; Published: 5 June 2017
}

In the published paper [1], there is an error in Figure 3. The red curve in Figure $3 b$ was deleted by mistake during the revisions. The correct figure should read as follows:

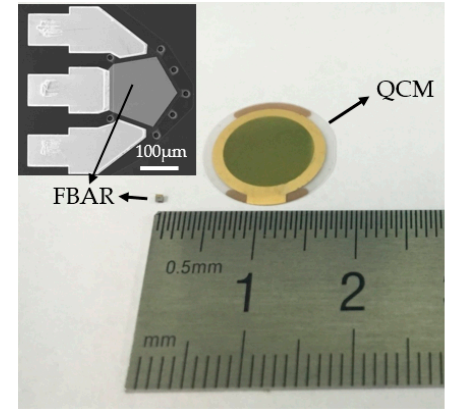

(a)

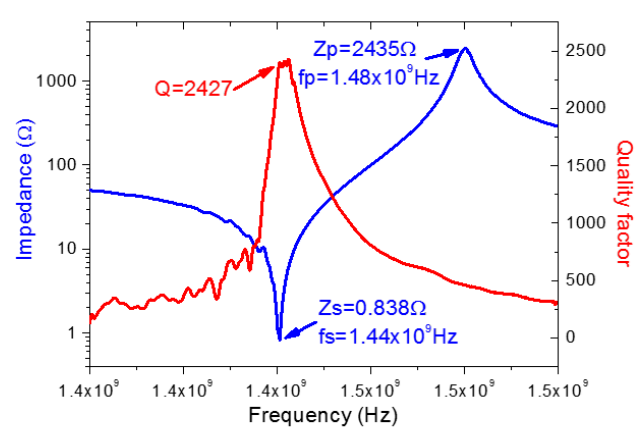

(b)

Figure 3. FBAR characterization. (a) A comparison of the size between FBAR and quartz crystal microbalance (QCM). The top-left inset shows the top-view scanning electron microscopy (SEM) image of FBAR; (b) magnitude of impedance and $Q$ value over frequency.

The authors apologize for any inconvenience caused by the error. The manuscript will be updated online and the previous version will remain available on the article webpage.

\section{Reference}

1. Liu, W.; Qu, H.; Hu, J.; Pang, W.; Zhang, H.; Duan, X. A Highly Sensitive Humidity Sensor Based on Ultrahigh-Frequency Microelectromechanical Resonator Coated with Nano-Assembled Polyelectrolyte Thin Films. Micromachines 2017, 8, 116. [CrossRef]

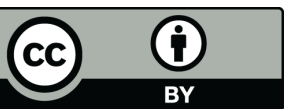

(C) 2017 by the authors. Licensee MDPI, Basel, Switzerland. This article is an open access article distributed under the terms and conditions of the Creative Commons Attribution (CC BY) license (http://creativecommons.org/licenses/by/4.0/). 